\title{
MicroRNA-1246 promotes growth and metastasis of colorectal cancer cells involving CCNG2 reduction
}

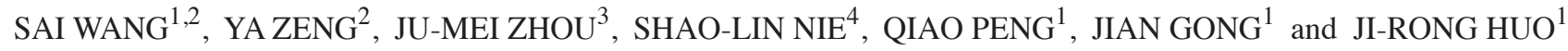 \\ ${ }^{1}$ Department of Gastroenterology, The Second Xiangya Hospital, Central South University; \\ ${ }^{2}$ Department of Digestive Diseases, Changsha Central Hospital; Departments of ${ }^{3}$ Radiotherapy and ${ }^{4}$ Colorectal Tumor Surgery, \\ The Affiliated Tumor Hospital of Xiangya Medical School of Central South University, Changsha, Hunan 410011, P.R. China
}

Received November 11, 2014; Accepted September 14, 2015

DOI: $10.3892 / \mathrm{mmr} .2015 .4557$

\begin{abstract}
Colorectal cancer (CRC) is the third most common cancer type and the fourth leading cause of cancer-associated mortality worldwide. MicroRNA (miR)-1246 is involved in differentiation, invasion, metastasis and chemoresistance of certain types of tumor cells. CCNG2 encodes an unconventional cyclin homolog, cyclin G2 (CycG2), associated with growth inhibition, which correlated significantly with lymph node metastasis, clinical stage, histological grade and poor overall survival in numerous cancer types. To investigate the regulation of $\mathrm{miR}-1246$ on $\mathrm{CycG} 2$ expression, and their effects on proliferation and metastasis of CRC, HCT-116 and LOVO cells were transfected with pre-miR-1246 anti-miR-1246 and their negative controls. It was demonstrated that the expression of miR-1246 was significantly increased in CRC tissues and cell lines, which was the opposite of CycG2. miR-1246 negatively regulated the expression of CycG2 in HCT-116 and LOVO CRC cells. CCNG2 is a direct target of miR-1246 in CRC cells. Overexpression of miR-1246 induced cell proliferation, migration and invasion, while knockdown of miR-1246 inhibited proliferation, migration and invasion in the CRC cells. Upregulation of miR-1246 mediated the malignant progression of CRC and is partly attributed to the downregulation of the expression of $\mathrm{CycG} 2$. Consequently, these findings provided a molecular basis for the role of miR-1246/CCNG2 in the progression of human CRC and suggested a novel target for the treatment of CRC.
\end{abstract}

Correspondence to: Professor Ji-Rong Huo, Department of Gastroenterology, The Second Xiangya Hospital, Central South University, 139 Middle Renmin Road, Changsha, Hunan 410011, P.R. China

E-mail: HJR198@hotmail.com

Key words: colorectal cancer, CCNG2, cyclin G2, miR-1246, metastasis

\section{Introduction}

Colorectal cancer (CRC) is the third most common cancer type and the fourth leading cause of cancer-associated mortality worldwide, with an estimated incidence of 1.2 million cases and a mortality of $>600,000$ annually ( $8 \%$ of all cancer-associated mortalities), making this a global public health problem. The incidence and mortality rates of CRC are increasing rapidly in Asian countries (1). Early-stage diagnosis of CRC potentially reduces the mortality of this disease. Although colonoscopic screening for CRC is currently the most reliable diagnostic tool, its cost and invasive nature limit its use. Therefore, there is a pressing requirement for developing novel, non-invasive, highly sensitive effective therapeutic methods (2).

MicroRNAs (miRs) belong to a class of highly conserved, 22-nucleotide, single-stranded RNAs, which epigenetically regulate protein translation by binding to the 3'-untranslated region (UTR) of the target messenger RNA (mRNA) and mediating either mRNA degradation or translational repression (3). miRs function as potential oncogenes or tumor suppressors in cancer development. A global impairment of miR has been described in various human cancer types, including CRC (4).

miR-1246 is a p53 transcriptional target, which is involved in the regulation of the known p53 functions, including cell cycle, apoptosis and senescence (5). The levels of serum miR-1246 can predict lymph node metastasis in cervical squamous cell carcinoma, which induces cervical squamous cell carcinoma $\mathrm{SiHa}$ cell proliferation, invasion and migration by targeting THBS2 $(6,7)$ and it may be a prognostic biomarker for oesophageal squamous cell carcinoma (8). miR-1246 is important in differentiation, invasion and metastasis of esophageal squamous cell carcinoma and hepatocellular carcinoma (9-11). miR-1246 was reported to be one of the driving miRs in CRC (12) since miR-1246 was significantly higher in tumor tissues compared with stromal tissues, and is present in higher serum exosomal levels in patients with CRC $(13,14)$. miR-1246 is considered to be responsible for proangiogenic function by activating Smad1/5/8 signaling in CRC (15). However, other biofunctions remain to be elucidated.

CCNG2 encodes an unconventional cyclin homolog, cyclin G2 (CycG2), associated with growth inhibition. The expression of $\mathrm{CycG} 2$ is repressed by mitogens, however, is upregulated during cell cycle arrest responses 
to antiproliferative signals (16). The expression of $\mathrm{CycG} 2$ decreased in thyroid cancer, esophageal, prostate, kidney and gastric cancer tissues and cells, and is significantly correlated with lymph node metastasis, clinical stage, histological grade and poor overall survival, suggesting that CCNG2 may be important as a negative regulator in thyroid cancer $\mathrm{K} 1$ cells by promoting the degradation of CDK2 (17-21). In addition, abnormal expression of $\mathrm{CycG} 2$ was observed in nasopharyngeal carcinoma cells and was closely associated with tumor differentiation, origin and progression (22). In colorectal carcinoma, the protein expression level of $\mathrm{CycG} 2$ was significantly lower in CRC tissue compared with normal tissues, and biological function revealed that SW480 cells transfected with CCNG2 exhibited a lower survival fraction, higher percentage of the $\mathrm{G} 0 / \mathrm{G} 1$ phases and reduced protein expression of CDK2 (21). The underlying molecular mechanism of the downregulation of $\mathrm{CycG} 2$ remains to be fully elucidated. In laryngeal squamous cell carcinoma, CCNG2 was confirmed as a direct target of miR-93 (23).

Notably, the expression of miR-1246 is associated with chemoresistance and cancer stem cell-like properties via CCNG2, and may predict a poorer prognosis in patients with pancreatic cancer (24). These results suggested that upregulation of miR-1246 may be one of the molecular mechanism of the regression of CCNG2 in pancreatic cancer cells. However, whether this is also the case in CRC cells remains to be elucidated.

The present study constructed cell models with gain-ofand loss-of-function of miR-1246 to assess its biofunction and direct target in CRC cell lines. These results may support CCNG2 and miR-1246 as novel diagnostic or therapeutic target for CRC.

\section{Materials and methods}

Ethics statement. Prior written informed consent was obtained from all patients and the study was approved by the Protection of Human Subjects Committee of the Second XiangYa Hospital of Central South University (Hunan, China).

Tumor tissue sample preparation. A total of 10 patients, including six males and four females aged 45-76 years (mean, 63.2 years), diagnosed with CRC at The Second XiangYa Hospital of Central South University were recruited. The experimental protocols were approved by the Ethics Committee of The Second XiangYa Hospital of Central South University. RNA or tissue samples were prepared from the tumor tissues and their matched adjacent non-tumor tissues, and were then subjected to reverse transcription-quantitative polymerase chain reaction (RT-qPCR) or western blotting.

Tissue microarray immunohistochemistry. A CRC tissue microarray (TC0128) was purchased from Auragene Biotechnology (Changsha, China). Tissue microarray blocks were incubated with anti-CCNG2 antibody (cat. no. ab5502; Abcam, Cambridge, MA, USA) or normal rabbit immunoglobulin $\mathrm{G}$ as a negative control. Immunostaining was performed using the Moticcam3000 system (Motic Group Co., Ltd., Xiamen, China) with diaminobenzidine (Zhongshan Jinqiao, Beijing, China). Image-Pro-Plus software (Media Cybernetics,
Inc., Rockville, MD, USA) was used to quantify the mean density of CCNG2 staining, according to the manufacturer's protocols.

Cell culture. The SW620, SW480, HCT116, HT29 and LOVO CRC cell lines, and intestinal epithelial cells (IECs) were purchased from China Center for Type Culture Collection (Wuhan, China). All cell lines were cultured in Dulbecco's modified Eagle's medium (Gibco; Thermo Fisher Scientific, Inc., Waltham, MA, USA), supplemented with $10 \%$ fetal bovine serum (Gibco; Thermo Fisher Scientific, Inc.), 100 IU/ml penicillin and $100 \mu \mathrm{g} / \mathrm{ml}$ streptomycin sulfate (Beyotime Institiute of Biotechnology, Shanghai, China) at $37^{\circ} \mathrm{C}$ in a humidified incubator, containing $5 \% \mathrm{CO}_{2}$.

Transfection. For the miR-1246 functional analyses, the pre-miR-1246, pre-con, anti-1246 or anti-con (GeneCopoeia, Guangdong, China) were transfected into HCT-116 and LOVO cell lines using Lipofectamine 2000 (Invitrogen; Thermo Fisher Scientific, Inc.), according to the manufacturer's protocols.

Reverse transcription-quantitative polymerase chain reaction (RT-qPCR). The total RNA was extracted from cells using TRIzol reagent (Invitrogen; Thermo Fisher Scientific, Inc.), according to the manufacturer's protocols. The relative expression level of miR-1246 and CCNG2 were determined by RT-qPCR using mirVana ${ }^{\mathrm{TM}}$ qRT-PCR microRNA detection kit (Ambion, Applied Biosystems, Austin, TX, USA), according to the manufacturer's protocols. The specific primer settings for miR-1246 (cat. no. HmiRQP0078) and U6 (cat. no. HmiRQP9003) were obtained from GeneCopoeia. The mRNA expression of CCNG2 was detected by RT-qPCR using the standard SYBR Green RT-PCR kit (Takara Bio, Inc., Otsu, Japan), according to the manufacture's protocols. The specific primer pairs were as follows: CCNG2, sense: 5'-AAGAAGAGAGATTCCAACC-3' and antisense: 5'-CCAGCAAAAAAGAACAGAC-3'; $\beta$-actin, sense: 5'-CCCATCTATGAGGGTTACGC-3' and antisense: 5'-TTTAATGTCACGCACGATTTC-3'. The mRNA expression levels were normalized against that of $\beta$-actin. The relative mRNA expression levels of CCNG2 or miR-1246 was quantified using the GraphPad Prism 4.0 software (GraphPad Software, San Diego, CA, USA) and the $2^{-\Delta \Delta C q}$ method (25).

Cell proliferation assay. Cells in the exponential growth phase were plated at a final concentration of $2 \times 10^{3}$ cells/well into 96-well culture plates. The viability of the cells was determined by MTT assay 24,48 and $72 \mathrm{~h}$ following seeding. The optical density at $570 \mathrm{~nm}$ of each well was measured using an ELISA reader (ELX-800; Bio-Tek, Winooski, VT, USA).

Colony-formation assay. For all groups, $3 \mathrm{ml}$ complete medium, containing 150 cells were added into each well of a 6-well plate. The plates were incubated at $37^{\circ} \mathrm{C}$ with $5 \% \mathrm{CO}_{2}$ for 14 days. Following incubation, the cells were gently washed and stained with Giems (Solabrio, Beijing, China). Colonies containing at least 50 cells $(0.3-1.0 \mathrm{~mm}$ in diameter) were counted using a microscope (AE31; Motic Group Co., Ltd.). 
Cell apoptosis assay. The cells were transfected with $80 \mathrm{nM}$ pre-miR-1246 or anti-miR-1246, and $48 \mathrm{~h}$ after transfection, the cells were subjected to an apoptosis assay. Apoptosis was detected by annexin V/propidium iodide staining using an apoptosis detection kit (BD Pharmingen, San Diego, CA, USA). Briefly, $10^{6}$ treated cells were incubated with annexin $\mathrm{V} /$ propidium iodide for $20 \mathrm{~min}$ at $25^{\circ} \mathrm{C}$ and were subsequently analyzed by flow cytometry (FACSCalibur; Beckman Coulter, Brea, CA, USA).

Cell invasion assay. The invasive ability of CRC cells was determined in 24-well Transwell chambers (Chemicon, Danvers, MA, USA), containing a layer of matrigel. For all groups, $200 \mu \mathrm{l}$ cell suspension $\left(1 \times 10^{6}\right.$ cells $\left./ \mathrm{ml}\right)$ was added in triplicate wells. Following an incubation for $24 \mathrm{~h}$, the dye on the membrane was dissolved with $10 \%$ acetic acid, dispensed into 96 -well plates $(150 \mu \mathrm{l} /$ well $)$ and the optical density at $570 \mathrm{~nm}$ of each well was measured using an ELISA reader (ELX-800; BioTek).

Cell migration assay. The cell migratory capability was estimated using a wound healing assay, as described previously (26). Briefly, the cells were cultured to confluence. Wounds of $\sim 1 \mathrm{~mm}$ width were created using a pipette tip of $1 \mathrm{ml}$, and the cells were washed and incubated in a serum-free medium. Following incubation for $24 \mathrm{~h}$, the cells were incubated in a medium, containing $10 \%$ fetal bovine serum. Cultures at 0 and $48 \mathrm{~h}$ were fixed and observed under a microscope (AE31; Motic).

Dual luciferase reporter assay. The 3'-UTR of CCNG2 (NM_004354.2), containing the miR-1246 binding sites and its corresponding mutated sequence, were cloned into the psi-CHECK2 luciferase reporter vector (Promega, Madison, WI, USA) downstream of Renilla luciferase, termed CCNG2-3'-UTR and CCNG2-Mut 3'-UTR, respectively. Using Lipofectamine 2000 (Invitrogen; Thermo Fisher Scientific, Inc.), the HCT-116 and LOVO cells were co-transfected with the recombinant reporter constructs and miR-1246 mimics, miR-1246 inhibitor, negative control (NC) or the negative control inhibitor (GeneCopopia, Rockville, MD, USA). Luciferase activity was determined after $48 \mathrm{~h}$ using the Dual-Glo substrate system (Promega) and an LD400 luminometer (Beckman Coulter). The data are presented as the ratio of experimental (Renilla) luciferase against control (Firefly) luciferase.

Western blotting. The tissues or cells were solubilized in cold radioimmunoprecipitation lysis buffer (Auragene, Changsha, China). Following lysis, a Bicinchoninic Acid Assay kit was used (Auragene) to determine protein concentration. The proteins $(2 \mu \mathrm{g} / \mu \mathrm{l})$ were separated on $10 \%$ SDS-PAGE gels (Amresco LLC, Solon, OH, USA) and were subsequently transferred onto a polyvinylidene membrane (EMD Millipore, Bedford, MA, USA). The membranes were blocked in $10 \%$ non-fat dried milk in phosphate-buffered saline containing $0.05 \%$ Tween-20 for $3 \mathrm{~h}$ and were subsequently incubated overnight at $4^{\circ} \mathrm{C}$ with rabbit anti-human CCNG2 polyclonal antibody (cat. no. ab5502; 1:1,000; Abcam), with $\beta$-actin (rabbit anti-human polyclonal antibody; 1:2,000; cat. no. 189073; Abcam) used as a control. Following primary

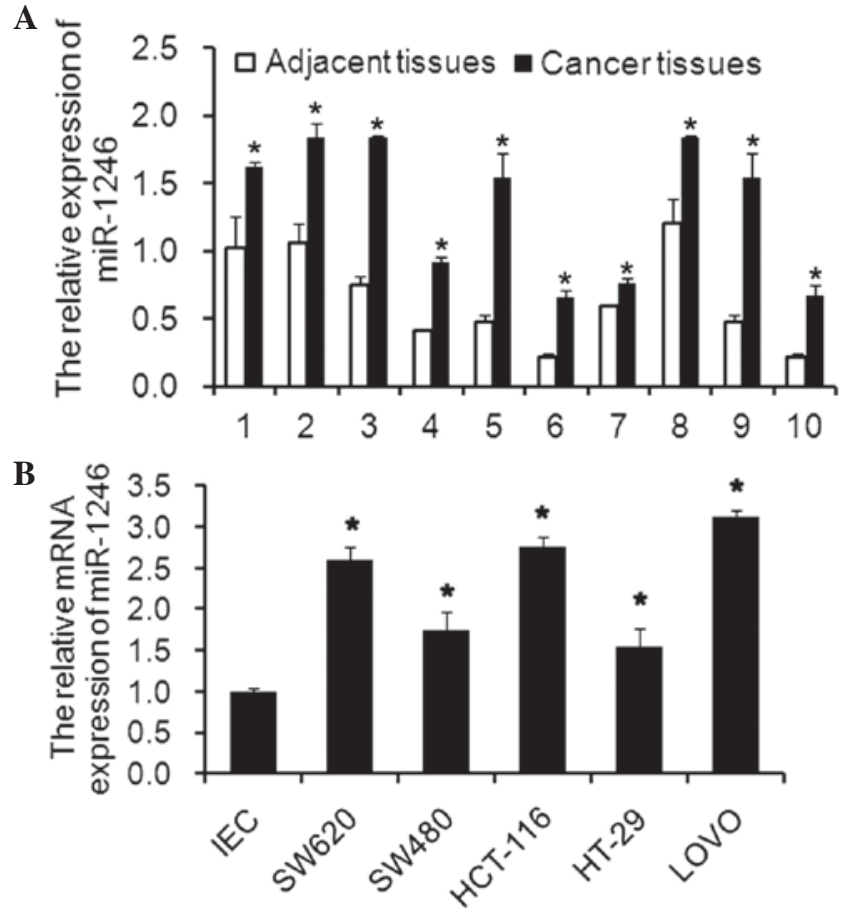

Figure 1. mRNA expression of miR-1246 in colorectal cancer. (A) The mRNA expression of miR-1246 was assessed in cancer tissues relative to the adjacent tissue from the identical patient and (B) the mRNA expression of miR-1246 was determined in five colorectal cancer cell lines relative to normal IEC cells by reverse transcription-quantitative polymerase chain reaction. The data are expressed as the mean \pm standard deviation of a representative experiment performed in triplicate. ${ }^{*} \mathrm{P}<0.05$. IEC, intestinal epithelial cell.

antibody incubation, the membrane was incubated with the goat-anti-rabbit-horseradish peroxidase secondary antibody (cat. no. 111-035-003; Jackson ImmunoResearch Laboratories, West Grove, PA, USA) at room temperature for $1 \mathrm{~h}$, and the immune complexes were detected using an enhanced chemiluminescence kit (Auragene).

Statistical analysis. The data are expressed as the mean \pm standard deviation from at least three separate experiments. Statistical analysis was performed using SPSS 16.0 software (SPSS, Inc., Chicago, IL, USA). The difference between two groups was analyzed by Student's t-test. $\mathrm{P}<0.05$ was considered to indicate a statistically significant difference.

\section{Results}

Expression of miR-1246 in CRC tissues and cell lines. The mRNA expression levels of miR-1246 in clinical tissues or in CRC cell lines were determined using RT-qPCR. Compared with the paired adjacent tissues, the mRNA expression levels of miR-1246 in the tumor tissues were significantly higher (Fig. 1A). Additionally, the mRNA expression levels of miR-1246 in SW620, SW480, HCT116, HT29 and LOVO cell lines were also upregulated compared with the levels in the IECs (Fig. 1B). The results suggested that miR-1246 may be an onco-miR involved in the progression and development of CRC.

Expression of CycG2 in CRC tissues and cell lines. CCNG2 was a predicted underlying target gene of miR-1246. 
A
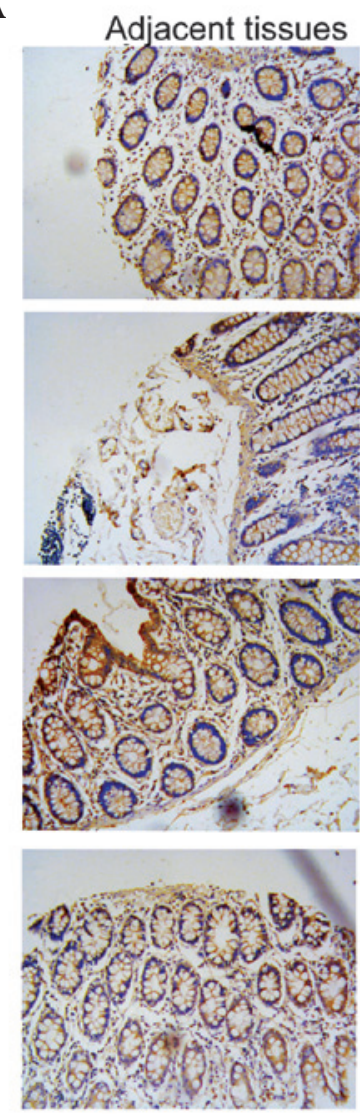

Cancer tissues

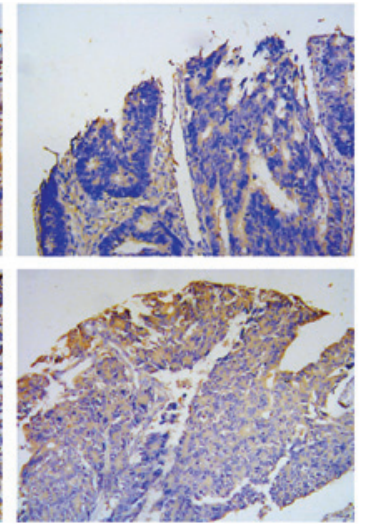

B

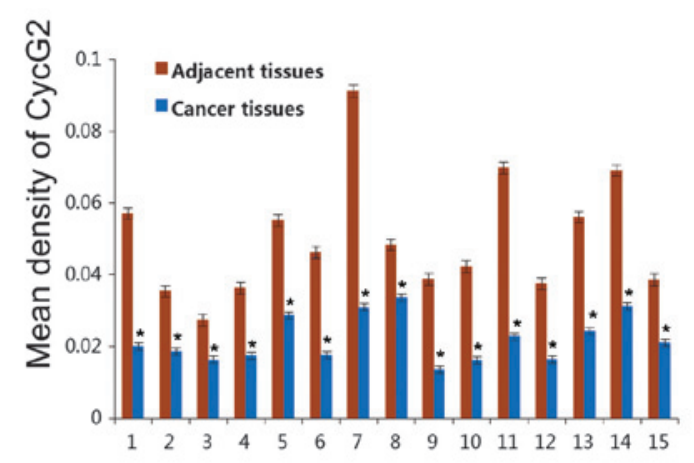

C
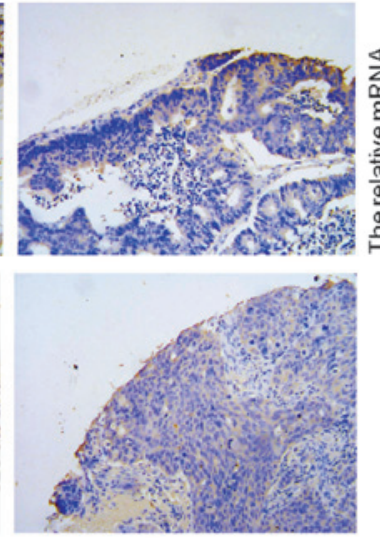

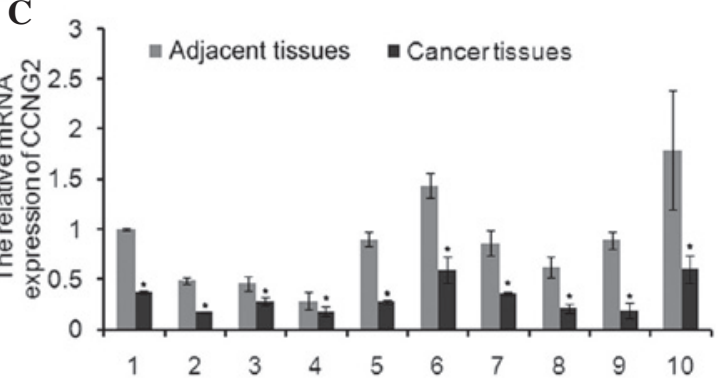

D

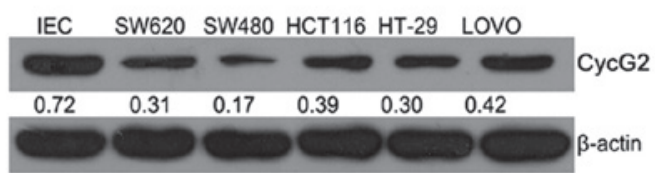

Figure 2. mRNA expression of CCNG2 and protein expression of CycG2 in colorectal cancer. (A and B) Representative results of immunohistochemical staining and mean density of $\mathrm{CycG} 2$ in cancer tissues and their paired adjacent tissues in the tissue microarray (magnification, $\mathrm{x} 40$ ). (C) The mRNA expression of CCNG2 in cancer tissues was determined relative to paired adjacent tissues by reverse transcription-quantitative polymerase chain reaction. (D) The protein expression of $\mathrm{CycG} 2$ in five colorectal cancer cell lines relative to endothelial IEC cells was determined by western blotting. The data are expressed as the mean \pm standard deviation of a representative experiment performed in triplicate. ${ }^{*} \mathrm{P}<0.05$, compared with the corresponding adjacent tissue group using Student's t-test. CycG2, cyclin G2; IEC, intestinal epithelial cell.

The tissue microarray analysis results demonstrated that the CycG2 mean density was significantly lower in tumor tissues compared with paired adjacent tissues (Fig. 2A and B).

The expression levels of $\mathrm{CycG} 2$ in collected CRC tissues and cell lines were assessed using RT-qPCR and western blotting. The results revealed that compared with the paired adjacent tissues, the mRNA expression levels of CCNG2 in tumor tissues were significantly lower (Fig. 2C) and the protein expression levels of CycG2 in the SW620, SW480, HCT116, HT29, LOVO cell lines were also downregulated compared with the IECs (Fig. 2D), which were consistent with the TMA results.

CCNG2 is a direct target of miR-1246 in the HCT-116 and LOVO cell lines. The coexistence of miR-1246 upregulation and CCNG2 downregulation in CRC tissues and cells implies a potential regulatory correlation between them. To confirm the direct targeting association between them, a luciferase reporter assay was used. The CCNG2 3'-UTR fragment, containing the miR-1246 binding site and its mutated sequence, were cloned into psi-CHECK 2 dual luciferase reporter vectors. miR-1246 significantly inhibited the luciferase activity in HCT-116 and LOVO cells transfected with the CCNG2-3'-UTR. However, miR-1246 mimics revealed no suppression on the luciferase activity levels in HCT-116 and
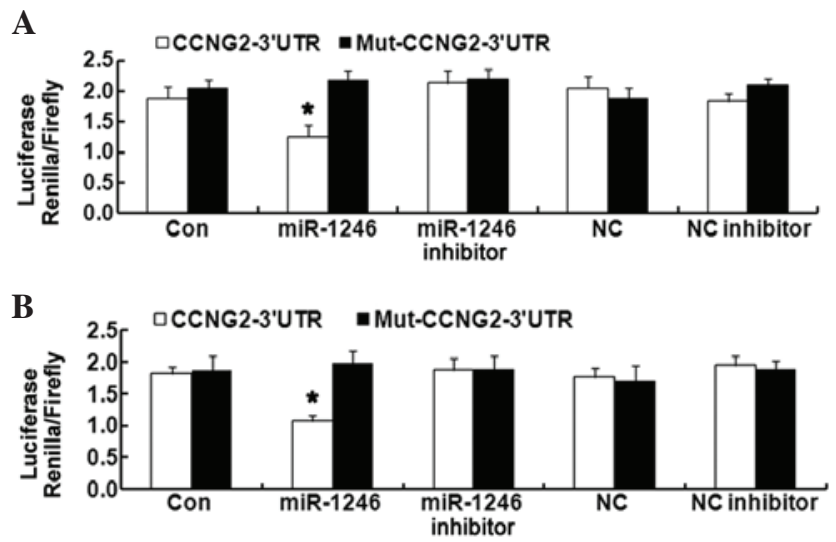

Figure 3. CCNG2 is a direct target of miR-1246. Dual luciferase reporter assays were performed to assess the interaction between miR-1246 and its targeting sequence in the CCNG2 3'-UTR, using constructs containing the targeting sequence (termed 3 '-UTR CCNG2) and mutated targeting sequence (termed 3'-UTR Mut CCNG2) cloned into psi-CHECK2 in (A) HCT-116 and (B) LOVO cells. The data are expressed as the mean \pm standard deviation of a representative experiment performed in triplicate $\left({ }^{*} \mathrm{P}<0.05\right.$, vs. NC). UTR, untranslated region; Mut, mutated; NC, negative control.

LOVO cells transfected with Mut-CCNG2-3'-UTR (Fig. 3). These results confirmed that $\mathrm{CCNG} 2$ is a direct target of miR-1246 in HCT-116 and LOVO cells. 
A

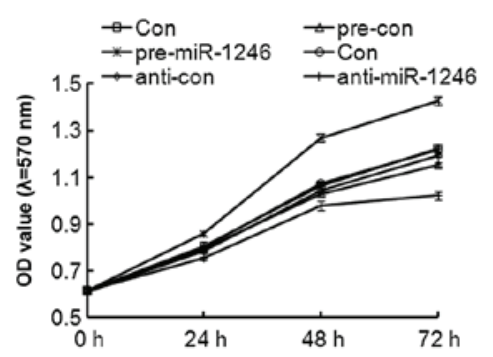

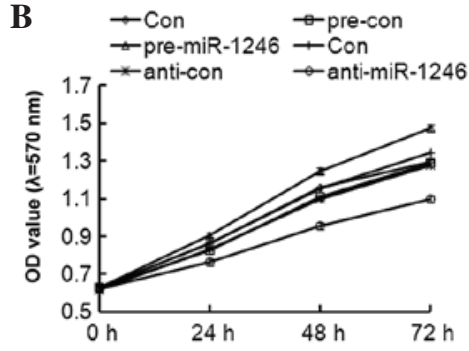

Figure 4. Effects of miR-1246 on cell proliferation. The colorectal cancer cells were transfected with pre-miR-1246, anti-miR-1246 or negative control (pre-con and anti-con). The cell viability between 0 and $72 \mathrm{~h}$ were measured using an MTT assay in (A) HCT-116 and (B) LOVO cells. Con, control; miR, microRNA; OD, optical density.

A

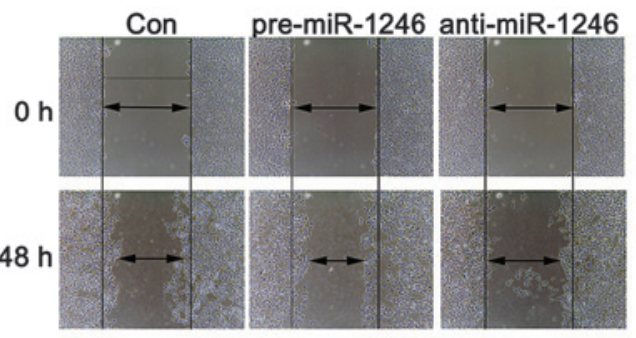

B

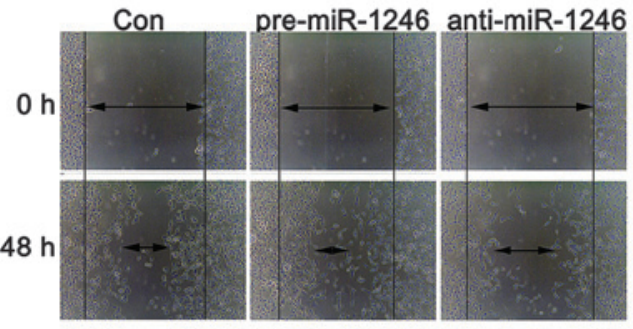

C
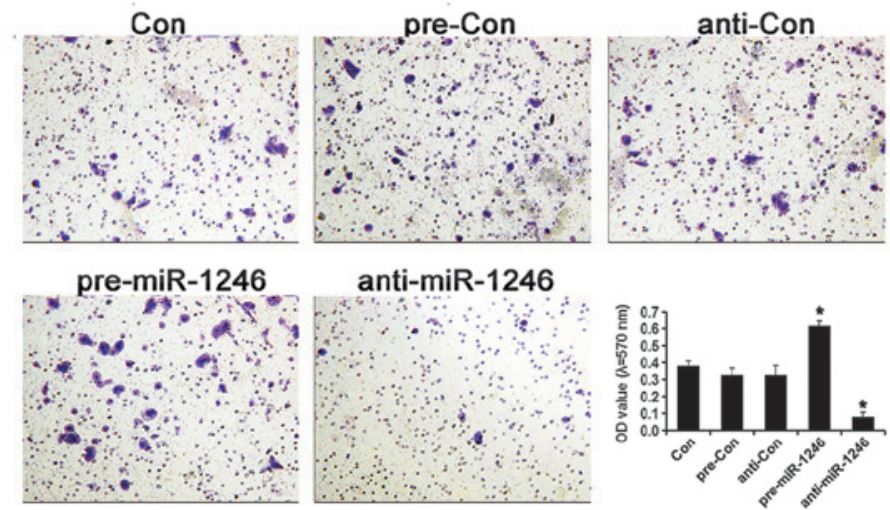

D
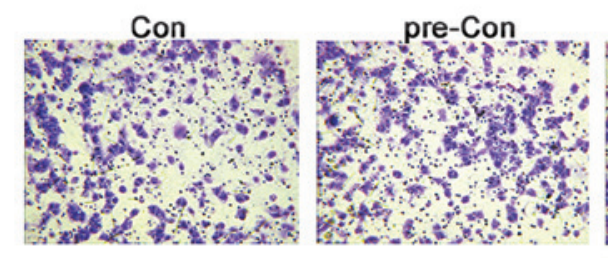

anti-miR-1246
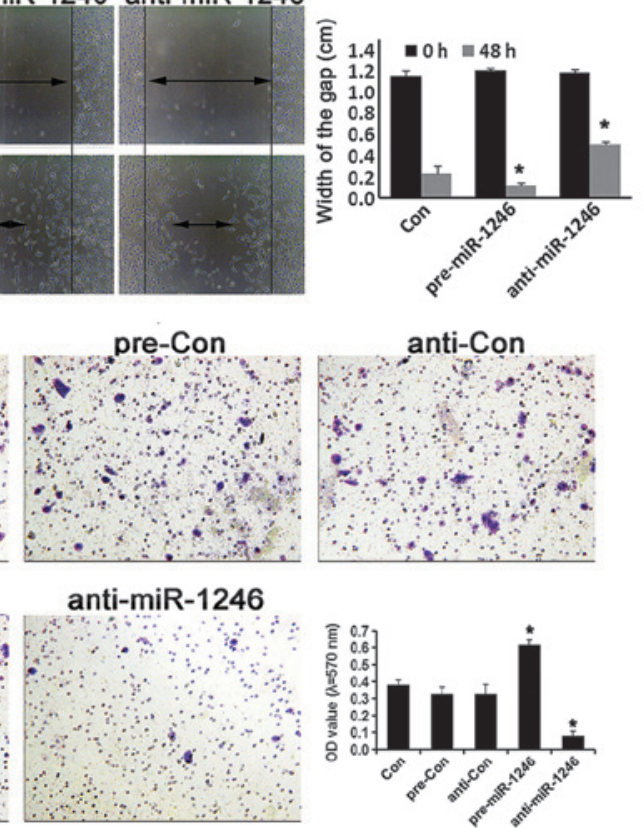

pre-miR-1246
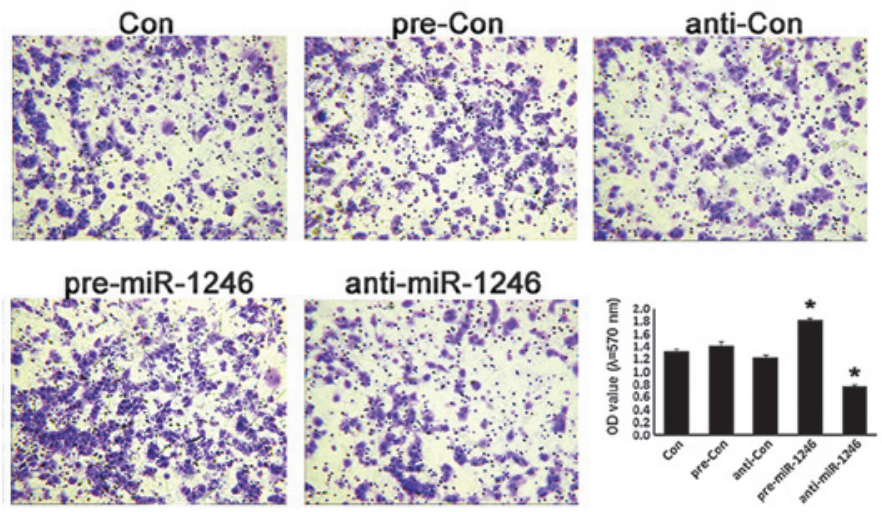

Figure 5. Effects of miR-1246 on cell invasion and migration. The migration capabilities were measured by scratch assay and the relative representative results were determined in (A) HCT-116 and (B) LOVO cells. The invasion capabilities were measured by Transwell assay and the relative representative results were determined in (C) HCT-116 and (D) LOVO cells. The data are expressed as the mean \pm standard deviation of a representative experiment performed in triplicate $\left({ }^{*} \mathrm{P}<0.05\right.$, vs. NC). NC, negative control; miR, microRNA; OD, optical density. 
A
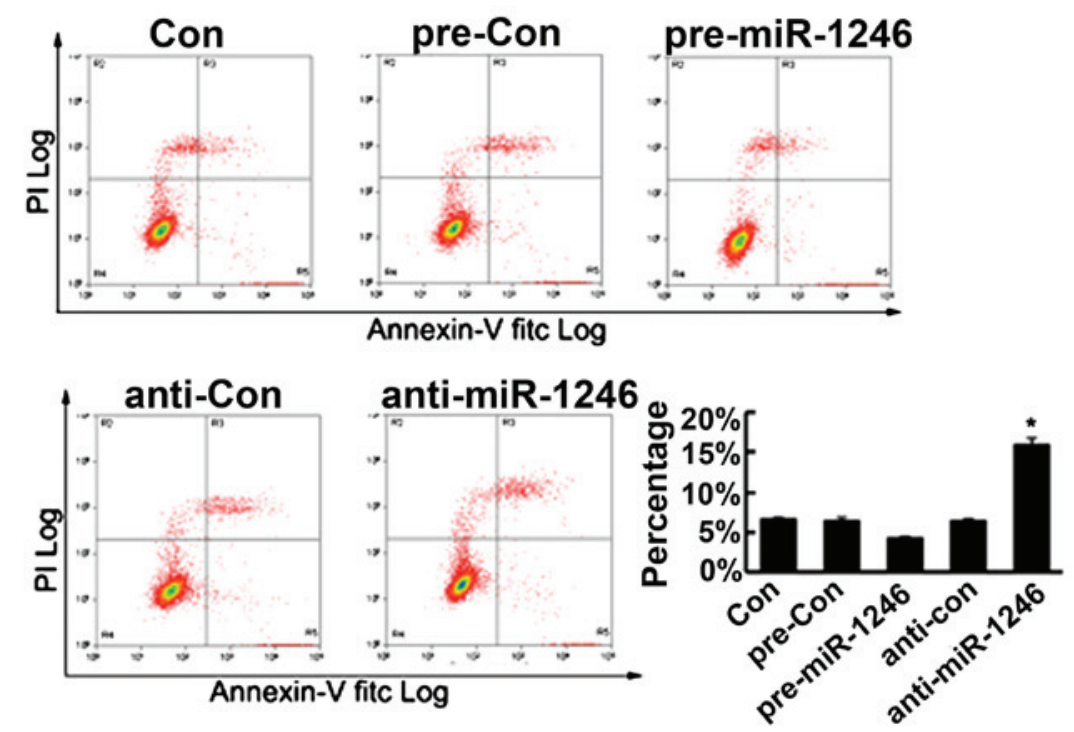

B

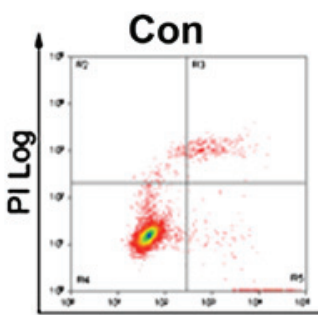

pre-Con
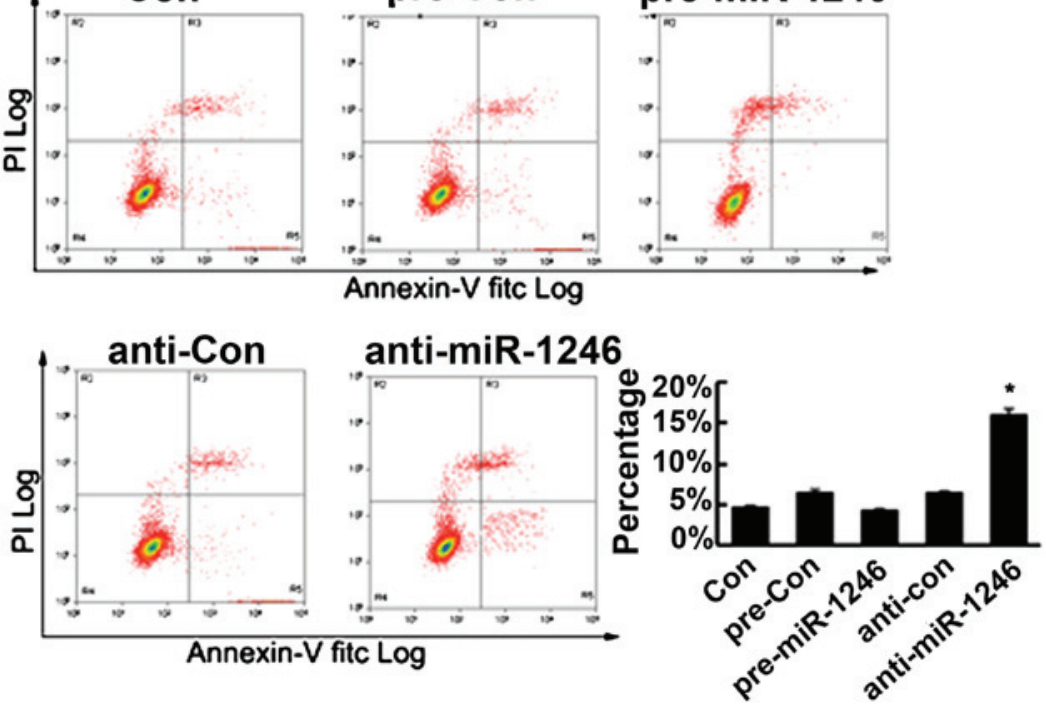

Figure 6. Effects of miR-1246 on cell apoptosis. (A) HCT-116 and (B) LOVO cells were transfected with pre-miR-1246, anti-miR-1246 or negative control (pre-con and anti-con) and apoptosis was assessed by annexin V-phycoerythrin/7-AAD flow cytometry. The error bars represent the mean \pm standard error of the mean ("P<0.05, vs. control). miR, microRNA.

Effects of miR-1246 on CRC proliferation. To further investigate the role of miR-1246 in CRC in vitro, a cell proliferation assay was performed to determine the effects of miR-1246 overexpression and knockdown on cell proliferation in HCT-116 and LOVO cells. Following transfection with pre-miR-1246, anti-miR-1246 and their negative control (pre-con and anti-con), RT-qPCR data revealed that the mRNA expression of miR-1246 was successfully altered. The expression of miR-1246 increased by almost 12 times in the HCT116 cell line, and 15 times in the LOVO cells, compared with the pre-con groups, following transfection with pre-miR-1246 transfection (data not shown). As shown in Fig. 4, the cell proliferation rate was even higher in the miR-1246-overexpressed HCT-116 and LOVO cells when compared with their parental or control groups; however, in miR-1246-knockdown HCT-116 and LOVO cells, the cell proliferation was reduced. These findings suggested that
miR-1246 has a positive role in HCT-116 and LOVO cell proliferation.

Effects of miR-1246 on CRC cell invasion and migration. The alternations of human CRC invasion were further investigated in miR-1246 gain- and loss-of-function HCT-116 and LOVO cells. As shown in Fig. 5, miR-1246-overexpression cells exhibited a higher invasive and migration capacity compared with cells in the control groups, while miR-1246-reduced cells exhibited a lower invasive phenotype and migration capacity compared with the relative controls $(\mathrm{P}<0.05$; Fig. 5). These data indicated that miR-1246 promoted the invasive ability and migration capacity in human CRC.

miR-1246 regulates cell apoptosis in CRC cells. To further determine the role of miR-1246 in CRC cells, cell apoptosis of the HCT-116 and LOVO cells was determined, subsequent to 
upregulating or downregulating miR-1246, using flow cytometry. As shown in Fig. 6, the upregulation of miR-1246 reduced cell apoptosis, however, this was not statistically significant. Conversely, the downregulation of miR-1246 significantly promoted cell apoptosis. Therefore, these findings confirmed that miR-1246 mediated the cell apoptosis in CRC cells in vitro.

\section{Discussion}

miR-1246 is known to be important in the progression of diverse cancer types. In the present study, it was revealed that miR-1246 was upregulated in the examined 10 tissues samples, which is consistent with previous reports $(5,8,27)$. These finding further illustrated that miR-1246 serves as an oncogene in CRC and its abnormal upregulation facilitates the progression of the tumor. It is well known that miRNAs function predominantly via special binding to the 3'-UTR of its target genes. Theoretical analysis suggests that the 3'-UTR of CCNG2 includes the binding sites of miR-1246. To confirm this, the HCT-116 and LOVO CRC cells were transfected with miR-1246 mimics or inhibitor. The results revealed that the protein expression levels of $\mathrm{CycG} 2$ were accordingly decreased or increased, suggesting that miR-1246 negatively regulated the expression of CCNG2 in CRC cells and that CCNG2 is the potential target gene of miR-1246. Subsequently, the dual luciferase reporter assay revealed that only miR-1246 mimics inhibited luciferase activity in HCT-116 and LOVO CRC cells transfected with 3'-UTR CCNG2 reporter vector. Therefore, for the first time, to the best of our knowledge, the present study demonstrated that CCNG2 is a target gene of miR-1246 in CRC cells.

The present study next investigated the potential effects of the miR-1246/CCNG2 pathway on HCT-116 and LOVO cells. The results revealed that the upregulation of miR-1246, which led to a decreased expression of $\mathrm{CycG} 2$, promoted the proliferation, colony formation, invasion and migration, and inhibited the apoptosis of CRC cells. However, downregulation of miR-1246 by its inhibitor, which led to increased expression of CycG2, inhibited these processes. These results suggested that miR-1246 upregulation may be critical in the malignant progression of CRC and its mechanism of action is partly involved in CCNG2.

The mechanisms underlying the function of miR-1246 as an inducer in CRC are implicated in promoting proliferation, invasion, migration, apoptosis and differentiation (for example, by suppressing THBS2 and CADM1) $(7,10)$. In the present study, certain of these molecules or others were possibly involved in the process of miR-1246 affecting growth and metastasis of HCT-116 and LOVO CRC cells. However, the present study hypothesized that CCNG2, as target of miR-1246, may also be involved in miR-1246 by promoting growth and metastasis of CRC cells. Previous studies indicated that CCNG2 is important for tumor cell proliferation. Downregulation of $\mathrm{CycG} 2$ can lead to growth enhancement in various cancer types. It was previously reported that downregulation of CCNG2 led to a reduced percentage of cells in the G0/G1 phases $(\mathrm{P}<0.05)$ and higher CDK2 protein expression in several types of tumor cell $(17,18,20)$. CCNG2 is also associated with invasion and lymph node metastasis, as confirmed in prostate cancer cells (19). These results confirmed that miR-1246 was a negative regulator of $\mathrm{CCNG} 2$ expression in $\mathrm{CRC}$ cells. Although the target gene of miR-1246 is not singular, it is logical to deduce that the upregulation of miR-1246, promoting proliferation, invasion, migration and inhibiting apoptosis in CRC cells is, at least partly, due to the downregulation of CCNG2.

miR-1246 was reported to be involved in stem cell characteristics, including chemoresistance and cancer stem cell-like properties via CCNG2 in pancreatic cancer (24), which may be an area of future work, to determine if miR-1246 influences the stemness of CRC stem cells and chemosensitivity of CRC cells.

In conclusion, miR-1246 was upregulated in CRC and negatively regulates the expression of CCNG2 in HCT-116 and LOVO CRC cells. miR-1246 upregulation mediated the malignant progression of $\mathrm{CRC}$, partly via the inhibition of CCNG2 expression. These findings provided a molecular basis for the role of miR-1246/CCNG2 in the progression of human CRC cells and suggested a novel target for the treatment of CRC.

\section{References}

1. Dong Y, Yu J and Ng SS: MicroRNA dysregulation as a prognostic biomarker in colorectal cancer. Cancer Manag Res 6: 405-422, 2014.

2. Yang Y, Gu X, Zhou M, Xiang J and Chen Z: Serum microRNAs: A new diagnostic method for colorectal cancer. Biomed Rep 1: 495-498, 2013.

3. Bartel DP: MicroRNAs: Genomics, biogenesis, mechanism and function. Cell 116: 281-297, 2004.

4. Lu J, Getz G, Miska EA, Alvarez-Saavedra E, Lamb J, Peck D, Sweet-Cordero A, Ebert BL, Mak RH, Ferrando AA, et al: MicroRNA expression profiles classify human cancers. Nature 435: 834-838, 2005.

5. Liao JM, Zhou X, Zhang Y and Lu H: MiR-1246: A new link of the p53 family with cancer and Down syndrome. Cell Cycle 11: 2624-2630, 2012

6. Chen J, Yao D, Li Y, Chen H, He C, Ding N, Lu Y, Ou T, Zhao S, Li L and Long F: Serum microRNA expression levels can predict lymph node metastasis in patients with early-stage cervical squamous cell carcinoma. Int J Mol Med 32: 557-567, 2013.

7. Chen J, Yao D, Zhao S, He C, Ding N, Li L and Long F: MiR-1246 promotes SiHa cervical cancer cell proliferation, invasion and migration through suppression of its target gene thrombospondin 2. Arch Gynecol Obstet 290: 725-732, 2014.

8. Takeshita N, Hoshino I, Mori M, Akutsu Y, Hanari N, Yoneyama Y, Ikeda N, Isozaki Y, Maruyama T, Akanuma N, et al: Serum microRNA expression profile: miR-1246 as a novel diagnostic and prognostic biomarker for oesophageal squamous cell carcinoma. Br J Cancer 108: 644-652, 2013.

9. Fu HL, Wu de P, Wang XF, Wang JG, Jiao F, Song LL, Xie H, Wen XY, Shan HS, Du YX and Zhao YP: Altered miRNA expression is associated with differentiation, invasion and metastasis of esophageal squamous cell carcinoma (ESCC) in patients from Huaian, China. Cell Biochem Biophys 67: 657-668, 2013.

10. Sun Z, Meng C, Wang S, Zhou N, Guan M, Bai C, Lu S, Han Q and Zhao RC: MicroRNA-1246 enhances migration and invasion through CADM1 in hepatocellular carcinoma. BMC Cancer 14: 616, 2014

11. Yan H, Wang S, Yu H, Zhu J and Chen C: Molecular pathways and functional analysis of miRNA expression associated with paclitaxel-induced apoptosis in hepatocellular carcinoma cells. Pharmacology 92: 167-174, 2013.

12. Piepoli A, Tavano F, Copetti M, Mazza T, Palumbo O, Panza A, di Mola FF, Pazienza V, Mazzoccoli G, Biscaglia G, et al: Mirna expression profiles identify drivers in colorectal and pancreatic cancers. PloS one 7: e33663, 2012.

13. Della Vittoria Scarpati G, Calura E, Di Marino M, Romualdi C, Beltrame L, Malapelle U, Troncone G, De Stefano A, Pepe S, De Placido S, et al: Analysis of differential miRNA expression in primary tumor and stroma of colorectal cancer patients. Biomed Res Int 2014: 840921, 2014 
14. Ogata-Kawata H, Izumiya M, Kurioka D, Honma Y, Yamada Y, Furuta K, Gunji T, Ohta H, Okamoto H, Sonoda H, et al: Circulating exosomal microRNAs as biomarkers of colon cancer. PloS One 9: e92921, 2014.

15. Yamada N, Tsujimura N, Kumazaki M, Shinohara H, Taniguchi K Nakagawa Y, Naoe T and Akao Y: Colorectal cancer cell-derived microvesicles containing microRNA-1246 promote angiogenesis by activating Smad 1/5/8 signaling elicited by PML down-regulation in endothelial cells. Biochim Biophys Acta 1839: 1256-1272, 2014.

16. Zimmermann M, Arachchige-Don AS, Donaldson MS, Dallapiazza RF, Cowan CE and Horne MC: Elevated cyclin G2 expression intersects with DNA damage checkpoint signaling and is required for a potent $\mathrm{G} 2 / \mathrm{M}$ checkpoint arrest response to doxorubicin. J Biol Chem 287: 22838-22853, 2012.

17. Chen JQ, Liu CJ, Wen HX, Shi CL, Zhang HS, Li M and Sun GG: Changes in the expression of cyclin G2 in esophageal cancer cell and its significance. Tumour Biol 35: 3355-3362, 2014.

18. Li WJ, Liu GL, Yu F, Xiang XX, Lu YF, Xiao HZ and Shi YP: CCNG2 suppressor biological effects on thyroid cancer cell through promotion of CDK 2 degradation. Asian Pac J Cancer Prev 14: 6165-6171, 2013.

19. Cui DW, Cheng YJ, Jing SW and Sun GG: Effect of cyclin G2 on proliferative ability of prostate cancer PC-3 cell. Tumour Biol 35: 3017-3024, 2014.

20. Cui DW, Sun GG and Cheng YJ: Change in expression of cyclin G2 in kidney cancer cell and its significance. Tumour Biol 35: $3177-3183,2014$
21. Sun GG, Zhang J and Hu WN: CCNG2 expression is downregulated in colorectal carcinoma and its clinical significance. Tumour Biol 35: 3339-3346, 2014.

22. Ye XX, Liu CB, Chen JY, Tao BH and Zhi-Yi C: The expression of cyclin $\mathrm{G}$ in nasopharyngeal carcinoma and its significance. Clin Exp Med 12: 21-24, 2012.

23. Xiao X, Zhou L, Cao P, Gong $H$ and Zhang $Y$ : MicroRNA-93 regulates cyclin G2 expression and plays an oncogenic role in laryngeal squamous cell carcinoma. Int J Oncol 46: 161-1741, 2015.

24. Hasegawa S, Eguchi H, Nagano H, Konno M, Tomimaru Y, Wada H, Hama N, Kawamoto K, Kobayashi S, Nishida N, et al: MicroRNA-1246 expression associated with CCNG2-mediated chemoresistance and stemness in pancreatic cancer. Br J Cancer 111: 1572-1580, 2014.

25. Arocho A, Chen B, Ladanyi M and Pan Q: Validation of the 2-DeltaDeltaCt calculation as an alternate method of data analysis for quantitative PCR of BCR-ABL P210 transcripts. Diagn Mol Pathol 15: 56-61, 2006.

26. Saadoun S, Papadopoulos MC, Hara-Chikuma M and Verkman AS: Impairment of angiogenesis and cell migration by targeted aquaporin-1 gene disruption. Nature 434: 786-792, 2005.

27. Pigati L, Yaddanapudi SC, Iyengar R, Kim DJ, Hearn SA, Danforth D, Hastings ML and Duelli DM: Selective release of microRNA species from normal and malignant mammary epithelial cells. PloS one 5: e13515, 2010. 